\title{
À propos du bicentenaire de Tocqueville
}

Ambiguïtés d'une redécouverte aux résonances multiples

Jacques Coenen-Huther

\section{(e) OpenEdition}

Journals

Édition électronique

URL : http://journals.openedition.org/ress/474

DOI : $10.4000 /$ ress. 474

ISSN : 1663-4446

Éditeur

Librairie Droz

\section{Édition imprimée}

Date de publication : 1 janvier 2006

Pagination : 153-162

ISBN : 2-600-00959-0

ISSN : 0048-8046

Référence électronique

Jacques Coenen-Huther, "À propos du bicentenaire de Tocqueville », Revue européenne des sciences sociales [En ligne], XLIV-133 | 2006, mis en ligne le 12 novembre 2009, consulté le 30 avril 2019. URL http://journals.openedition.org/ress/474; DOI : 10.4000/ress.474 
Jacques COENEN-HUTHER

\section{A PROPOS DU BICENTENAIRE DE TOCQUEVILLE Ambiguités d'une redécouverte aux résonances multiples}

Dans les premières années du $\mathrm{XX}^{\mathrm{e}}$ siècle, un commentateur de l'œuvre tocquevillienne écrivait: «Malgré ses mérites, Tocqueville est presque abandonné» (Pierre-Marcel, 1910, p. 457). En l'année du bicentenaire de sa naissance ${ }^{1}$, marquée par un foisonnement de colloques et de publications, c'est un Tocqueville consacré qui a fait place au Tocqueville oublié d'autrefois, avec tous les effets de mode que recèle ce renouveau d'intérêt. Loin d'être le résultat d'un consensus réalisé sur son œuvre, cette redécouverte chargée d'ambiguïtés reflète la convergence apparente de préoccupations très diverses. Tocqueville, réexhumé par Raymond Aron dans les années 1950, c'est avant tout le penseur critique d'une société de classes moyennes accédant à la prospérité généralisée et s'écartant toujours davantage des schémas marxistes. Tocqueville, invoqué par Michel Crozier dans les années 1960, c'est l'analyste de la machine bureaucratique française et de sa lourdeur centralisatrice. Dans la décennie qui suit, c'est le théoricien de la continuité historique dans le très long terme qui séduit. François Furet confère à l'Ancien Régime et la Révolution une importance décisive pour la compréhension de l'histoire de France. Dans le même temps, Raymond Boudon découvre en Tocqueville une figure emblématique de l'individualisme méthodologique et le présente comme un précurseur de Max Weber ${ }^{2}$. Ces divergences ne se sont pas estompées, bien au contraire. C'est ce qui fait dire à Serge Audier, auteur d'un passionnant Tocqueville retrouvé ${ }^{3}$, qu'on trouve «des références à Tocqueville dans une multitude d'écrits relevant de préoccupations extrêmement différentes » (2004, pp. 8-9).

En guise d'introduction à un important colloque international consacré à «Alexis de Tocqueville entre l'Europe et les Etats-Unis» organisé à Cerisy-laSalle en mai $2005^{4}$, Jean-Claude Casanova, directeur de la revue Commentaire, faisant écho à Pierre-Marcel, a rappelé la période d'indifférence à l'œuvre de Tocqueville qui s'est étendue en France du début de la Première Guerre mondiale aux lendemains de la seconde. Pour Casanova, dans l'entre-deux guerres, seul Elie Halévy, avec ses réflexions sur le socialisme, sur l'Etat-nation et sur la guerre,

\footnotetext{
${ }^{1}$ Alexis de Tocqueville naquit le 29 juillet 1805.

${ }^{2} \mathrm{Au}$ sujet de cette succession de figures diverses, voir mon Tocqueville (Paris, PUF, Coll. «QSJ?», 1997, p. 123).

${ }^{3}$ Dont il sera question plus loin.

${ }^{4}$ Dont on rend compte ici de manière très sélective mais dont les actes feront l'objet d'une publication.
} 
se situe dans la continuité de Tocqueville. Le colloque de Cerisy ouvert par Casanova bénéficia de la participation de spécialistes américains et français des études tocquevilliennes, entre autres Jean-Louis Benoît, Pierre Manent et Françoise Mélonio pour la France, David Bell, Seymour Drescher et James Schleifer pour les Etats-Unis. Des lectures divergentes de l'œuvre de Tocqueville s'y côtoyèrent inévitablement; de part et d'autre de l'Atlantique, on ne privilégie pas toujours les mêmes thèmes. Si en France, les discussions se centrent volontiers sur la dimension libérale de la pensée de Tocqueville (Pierre Manent), aux Etats-Unis le thème cultivé le plus volontiers est celui de la participation politique et du self-government sur le plan local (Cheryl Welch, Boston; Jeffrey Alexander, Yale). Mais dans les deux cas, on n'échappe pas à la question de la transfiguration possible du passé. S'agissant de l'Amérique, c'est la pertinence actuelle de Tocqueville qui semble au centre des interrogations. Il y a lieu en effet de se demander si une description des Etats-Unis datant d'avant la Guerre de Sécession garde un quelconque intérêt de nos jours. A ce sujet, on se doit pourtant de rappeler que les jugements portés par Tocqueville sur le sort de la population indienne et sur celui des Noirs avaient à l'époque un caractère prémonitoire. Il faut également relativiser la rapidité du changement social et politique dans la société nord-américaine. A l'encontre des notions convenues sur la jeunesse et le dynamisme des Etats-Unis, on prend actuellement conscience de l'ancienneté et de la stabilité des institutions américaines. Comme le fait observer Anatol Lieven dans un ouvrage récent, ces institutions - à commencer par la Constitution américaine qui date de 1787 - sont plus anciennes et ont moins changé que celles de la plupart des autres Etats du monde (Lieven, 2004, p. 51). Pour ce qui est de la France, c'est la réalité historique des relations sociales d'Ancien Régime vues par Tocqueville qui suscite des interrogations. A Cerisy, Françoise Mélonio n'hésita pas à affirmer que Tocqueville s'est «inventé une aristocratie» et que les relations de confraternité entre seigneurs et paysans relèvent pour une part d'un «passé idéalisé». Les sociologues y voient plutôt une manière idéal-typique d'opposer le monde de la tradition à la modernité.

Le colloque de Cerisy fut également le lieu d'intéressantes convergences. Regina Pozzi (Pisa) et David Bell (Johns Hopkins) traitèrent tous deux de la continuité historique chez Tocqueville. A l'encontre de Burke qui attribuait la destruction du tissu social de l'Ancien Régime au choc brutal de la Révolution française, Tocqueville y voyait le résultat d'une action politique entamée par les rois de France (Pozzi). Ce rôle politique de la monarchie s'illustre notamment par le véritable coup d'Etat entrepris par Louis XV contre les parlements et qui préfigure en quelque sorte celui de Louis Napoléon, suggérant ainsi un parallélisme entre les années 1770 et les années 1850 (Bell). On s'accorde actuellement à penser que la figure tragique de Malesherbes, bisaïeul de Tocqueville, contribua à inspirer à ce dernier le sens profond de la continuité entre l'Ancien Régime et la modernité, audelà de la césure révolutionnaire. Dans cette perspective, l'action centralisatrice des rois de France amorce donc l'affaiblissement durable des pouvoirs intermédiaires. A ce sujet aussi, il faut mettre en garde contre toute idéalisation du passé. Stephen Smith (Yale) fit observer que les pouvoirs intermédiaires constituaient la base de pouvoir de l'aristocratie et que la centralisation s'inscrivait dans la dialectique liberté-égalité qui domine l'œuvre tocquevillienne.

La vision continuiste de Tocqueville permet d'établir un lien entre les conceptions aristocratiques et le libéralisme politique moderne. On peut, comme j'ai eu 
l'occasion de le faire, mettre l'accent sur la passion de la liberté qui anime Tocqueville (Coenen-Huther, 1997, p. 16). C'est en effet la liberté comme valeur ultime qui livre le secret de sa pensée et lui confère sa cohérence. Mais ses hésitations, ses déchirements, ses contradictions apparentes se comprennent mieux à la lumière d'une analyse de la notion de liberté telle qu'elle apparaît dans ses raisonnements. Pour Alan Kahan (Florida International University), Tocqueville est aux prises avec deux conceptions différentes de la liberté: la liberté aristocratique, basée sur des privilèges, et la liberté démocratique, fondée sur des droits universels. Dans le contexte du monde démocratique qu'il a décidé d'accepter, il ne peut s'empêcher de vouloir préserver certains traits de la société aristocratique. Françoise Mélonio distingue chez Tocqueville, trois acceptions du terme «liberté »: la liberté du libéralisme politique qui existe grâce aux garanties contre les abus de pouvoir, la liberté républicaine qui repose sur la participation à la chose publique, enfin la liberté morale qui se nourrit de l'indépendance d'esprit de l'individu. Dans tout l'œuvre, il y a oscillation entre ces différentes conceptions de la liberté. L'attention portée aux conditions sociales de la liberté est plus ou moins forte selon la conception de la liberté qui est prise en compte.

Plusieurs intervenants au Colloque de Cerisy mirent en évidence la figure de Tocqueville homme politique, qui tend à être négligée par les exégètes récents. Pour Robert Gannett (Chicago), la présentation unilatérale de Tocqueville comme philosophe politique et comme théoricien correspond à une tendance nouvelle. Il est également un homme politique orienté vers l'action, qui a gagné plusieurs élections consécutives. En dépit de sa réputation d'inefficacité ${ }^{5}$, il a pu se prévaloir de succès remarquables dans sa circonscription. C'était un homme passionné, prêt à prendre des risques en politique. Seymour Drescher (Pittsburgh) intervint dans le même sens en indiquant que Tocqueville s'était de toute évidence donné pour tâche d'exercer une influence sur l'avenir de son pays.

L'orientation libérale de Tocqueville fit l'objet d'interventions en sens divers. Pierre Manent (EHESS, Paris) présenta très classiquement Tocqueville comme un philosophe politique libéral. Mais la «science politique nouvelle» dont celui-ci se fait l'avocat dans la première Démocratie n'est autre, selon Manent, que la science politique libérale de Montesquieu. Ce libéralisme-là, il faut le souligner, est un libéralisme politique et intellectuel qui ne suffit pas à justifier la présentation de Tocqueville comme un précurseur du libéralisme économique moderne. Eric Keslassy (Paris-Dauphine) fit un pas de plus et émit l'hypothèse que si l'aspect «social » de la pensée de Tocqueville est négligé, c'est précisément parce que ce sont des libéraux - au sens français moderne du terme - qui se sont chargés de diffuser son œuvre. En fait, et l'on en conviendra avec Keslassy, Tocqueville était préoccupé par le paupérisme, à savoir la «nouvelle pauvreté», la pauvreté de masse engendrée par l'essor de l'économie capitaliste. Il était hostile à l'idée d'un Etat entrepreneur mais il souscrivait à la nécessité d'un Etat régulateur. En faire un précurseur de Hayek serait trahir sa pensée. Jean-Louis Benoît (Université de Caen) abonda dans le même sens. Pour lui, si Tocqueville est sensible à l'idée de charité chrétienne, il est conscient du fait que cette charité est devenue inopérante

\footnotetext{
${ }^{5}$ Que lui valut surtout son bref passage au Ministère des Affaires Etrangères dans le second cabinet Odilon Barrot (JCH).
} 
face au paupérisme. Il est hostile aux mesures qui lui paraissent relever de l'assistance sociale généralisée mais il est d'avis qu'il y a pour l'Etat un devoir d'intervention sociale. Il est favorable à l'élaboration d'un cadre législatif pour la lutte contre le paupérisme et il s'intéresse au régime fiscal dans le souci d'aider les défavorisés du sort. Les mesures de politique sociale préconisées par Tocqueville sont celles que recommandaient les «philanthropes » d'orientation sociale-chrétienne de l'époque.

Si l'année du bicentenaire aura été célébrée par une série de colloques - à Cerisy-la-Salle mais aussi à la Sorbonne, à l'Université de Caen, à Yale ou à Bruxelles - et par une passionnante exposition intitulée "Qui êtes-vous Monsieur de Tocqueville?» aux Archives départementales de la Manche, à Saint-Lô ${ }^{6}$, son approche a également suscité une floraison de publications nouvelles. Certes, deux ouvrages continuent à faire autorité: la belle biographie d'André Jardin, Alexis de Tocqueville 1805-1859 (1984), qui vient de faire l'objet d'une réimpression dans la collection de poche «Pluriel» (2005) et l'excellent ouvrage de Françoise Mélonio, Tocqueville et les Français (1993), qui retrace les étapes de la réception de Tocqueville en France. Par ailleurs, le Tocqueville de la collection «Bouquins» (1986, réimpressions 1991 et 1999) reste un instrument de travail très pratique. Il n'en reste que les études tocquevilliennes, comme on va le voir, se sont considérablement enrichies au cours de ces dernières années.

Signalons tout d'abord une nouvelle biographie de Tocqueville par Jean-Louis Benoît sous le titre Tocqueville. Un destin paradoxal (2005). Par rapport à la biographie d'André Jardin, l'imposant ouvrage de Benoît est centré davantage sur la personne de Tocqueville et moins sur son environnement social; il contient plus de détails sur les différents épisodes de sa vie et sur ses relations avec ses proches. On y trouve de nombreuses mises en relation avec des faits contemporains sans que l'œuvre tocquevillienne en soit pour autant décontextualisée. Benoît souligne le «volontarisme éthique et politique» de Tocqueville (2005, p. 329). Au passage, il fustige les «contresens » qui ont fini par constituer « la vulgate tocquevillienne » ainsi que les «lectures partielles, indigentes et surprenantes d'un auteur d'importance majeure » (ibid., p. 137). En somme, la biographie d'un homme engagé, écrite par un homme engagé! Auparavant, Benoît s'était déjà signalé par une anthologie, Alexis de Tocqueville, textes essentiels (2000), et plus récemment, coup sur coup, par trois publications dignes d'attention: Comprendre Tocqueville, ouvrage à visée didactique dans la collection «Cursus» d'Armand Colin (2004), Tocqueville moraliste, un livre dans lequel la dimension morale de l'œuvre tocquevillienne apparaît grâce à l'analyse des textes consacrés au paupérisme, à la réforme pénitentiaire ainsi qu'aux Indiens d'Amérique et aux esclaves noirs des Etats Unis (2004); enfin, en collaboration avec Eric Keslassy, Alexis de Tocqueville. Textes économiques (2004), où Tocqueville apparaît préoccupé «des implications de l'économie dans la sphère sociale et politique» (ibid., p. 17). Quelques années plus tôt, le collaborateur de Jean-Louis Benoît, Eric Keslassy, avait publié Le libéralisme de Tocqueville à l'épreuve du paupérisme (2000), ouvrage dans lequel, comme l'indique Françoise Mélonio dans sa préface, se

\footnotetext{
${ }^{6}$ Exposition à laquelle est associé un superbe album qui constitue bien davantage qu'un catalogue (Charlotte Manzini, 2005)
} 
profile un Tocqueville manifestant sa volonté d'être «un libéral d'une espèce nouvelle » et cherchant «une troisième voie entre socialisme et libéralisme économique», à savoir «la voie d'une société solidaire» (Mélonio, in Keslassy, 2000, p. 15$)^{7}$.

Laurence Guellec, qui avait déjà publié un petit ouvrage intitulé Tocqueville. L'apprentissage de la liberté en 1996, a donné aux éditions Champion un Tocqueville et les langages de la démocratie (2004). Elle y recommande d'analyser l'ensemble de l'œuvre tocquevillienne «comme un texte argumentatif». On échappe ainsi, estime-t-elle, à ce qu'elle appelle plaisamment «l'Arlésienne des sociologues », à savoir « les débats absurdes sur la scientificité ou la non-scientificité de l'introuvable méthode tocquevillienne» (Guellec, 2004, pp. 19-20 et note 24). Tocqueville était en effet un publiciste qui écrivait pour les notables constituant la classe politique de son temps, avec la volonté affirmée d'influencer l'opinion. Il serait absurde d'en faire a posteriori un méthodologue. Tout ce qu'on peut en dire - mais cela témoigne de la dimension sociologique de son œuvre - c'est que ses textes livrent une méthodologie implicite qu'il est possible de faire apparaître. Et cet exercice est loin d'être futile car il illustre des enjeux majeurs d'épistémologie sociologique ${ }^{8}$.

Agnès Antoine, dans L'impensé de la démocratie. Tocqueville, la citoyenneté et la religion (2003), indique clairement sa volonté de considérer Tocqueville «comme un philosophe à part entière » $(2003$, p. 15), à savoir un philosophe «du mal-être de la modernité » (ibid., p. 336). Mais si Agnès Antoine présente Tocqueville comme un moraliste et un métaphysicien, s'interrogeant, à travers l'analyse du processus d'évolution démocratique, sur «le sort futur de l'espèce humaine» (ibid., p. 9), elle n'en restitue pas moins l'esprit sociologique qui imprègne son œuvre. Dans le monde aristocratique - et ceci rend compte de la nostalgie de l'Ancien Régime qui perce sous l'adhésion raisonnée de Tocqueville à l'ordre démocratique - la hiérarchie des différents ordres «implique la clarté et la réciprocité des obligations morales ». La société égalitaire, «par son principe même» dont il serait vain de vouloir nier le caractère équitable, «crée de la déliaison» (ibid., pp. 9-10). C'est ici qu'intervient la relation entre citoyenneté et religion annoncée par le titre du livre. La société inégalitaire d'Ancien Régime implique la référence à une réalité transcendante qui lui confère du sens, elle reflète «la soumission à un ordre à la fois antérieur et extérieur de nature divine». Dans l'ordre démocratique laïcisé, «les hommes désormais déliés» sont voués à «définir eux-mêmes leur propre norme », avec les risques de «relativisme généralisé» et de dégradation de l'éthique démocratique que cela comporte (ibid., pp. 10-11).

Dans Tocqueville retrouvé (2004), Serge Audier s'interroge sur le sens de ce renouveau tocquevillien français dont les symptômes se multiplient. Le titre de l'ouvrage reproduit celui d'un article de Raymond Aron, consacré au retour de Tocqueville sur la scène intellectuelle et politique française (Aron, 1979). L'auteur montre cependant que la redécouverte de Tocqueville ne saurait être attribuée

\footnotetext{
${ }^{7}$ Eric Keslassy vient de soutenir avec succès à l'université Paris IX-Dauphine une thèse de doctorat en sociologie intitulée "Question sociale et démocratie dans l'œuvre de Tocqueville», préparée sous la direction du Professeur Philippe Steiner (Keslassy, 2005).

${ }^{8}$ Je me permets à ce sujet de renvoyer à nouveau à mon «Que sais-je?» consacré à Tocqueville (1997, pp. 4-5).
} 
uniquement aux efforts d'Aron. Les diverses relectures auxquelles on a fait allusion plus haut reflètent bien des préoccupations d'ordres différents et prennent appui sur des interprétations antérieures dont il n'est pas sans intérêt d'établir la généalogie. En regard du Tocqueville dont on a opposé l'héritage intellectuel à celui de Comte et de Marx, il y a le Tocqueville anti-jacobin de Sorel, le Tocqueville anti-démocrate de Rédier ou encore le Tocqueville annonciateur d'un despotisme nouveau de J.P. Mayer. Il y a également l'inspirateur de Célestin Bouglé et de Louis Dumont. Pour Audier, la redécouverte de Tocqueville est loin d'être un phénomène homogène; il s'agit d'un processus complexe, dominé par les conflits d'interprétation. En France, la tendance dominante est à cataloguer Tocqueville comme un libéral, au prix d'une confusion entre le libéralisme politique et le libéralisme ou néo-libéralisme économique. Aux Etats-Unis, on insiste sur la dimension républicaine de sa pensée, privilégiant le thème de la participation politique. Les commentateurs du renouveau tocquevillien se partagent entre ceux qui ne veulent y voir qu'un phénomène idéologique et ceux qui en font le signe d'une évolution de la pensée politique française. Audier rejette autant les lectures nostalgiques anti-modernes de l'œuvre tocquevillienne que les interprétations qui la réduisent à une apologie de la modernité libérale et démocratique. Tocqueville est un libéral à la manière de Montesquieu pour qui la société d'un peuple libre est une société toujours en mouvement, nécessairement traversée par des tensions. Ces tensions, Tocqueville n'a pas cessé de les vivre personnellement. En ce sens, selon Audier, le libéralisme de Tocqueville est un libéralisme post-machiavélien. Car il est tout à fait possible de combiner la vision de l'évolution démocratique avec la lucidité à l'égard du rôle des élites'.

Contrairement à la plupart des auteurs précédents, c'est un Tocqueville volontairement décontextualisé que nous propose Raymond Boudon avec son Tocqueville aujourd' hui, paru chez Odile Jacob (2005). L'objectif du livre est de montrer l'actualité de Tocqueville en le présentant comme un classique de la sociologie, soucieux d'élaboration théorique et de méthode, dont les analyses conservent toute leur pertinence de nos jours. Il est vrai que nombre des argumentations de Tocqueville, détachées de leur contexte d'origine, préfigurent les théories sociologiques les plus solides. Il est vrai aussi que ses procédés d'analyse évoquent une méthode implicite qu'il est intéressant de faire apparaître. Mais sa préoccupation première n'était pas d'ordre théorique ou méthodologique. Il avait le souci d'influencer l'opinion et l'esprit sociologique qui l'animait n'était pas prioritairement au service d'un projet scientifique. Comme d'autres pionniers de la sociologie, Tocqueville fut sans cesse tiraillé entre les exigences de la vie intellectuelle et celles de l'engagement dans les affaires de la cité. Le discours de 1852 à l'Académie des sciences morales et politiques était certes un plaidoyer pour les sciences sociales dont Tocqueville ne doutait pas qu'elles pouvaient être des sciences au sens plein du terme. Raymond Boudon a raison de le souligner (2005, p. 13). Mais ce discours était aussi un testament politique au sortir de la vie publique et le discours de combat d'un «exilé de l'intérieur » ${ }^{10}$. Tocqueville y défend la liberté

\footnotetext{
9 Voir à ce sujet ma Sociologie des élites (2004, notamment pp. 25-26).

${ }^{10}$ On consultera utilement à ce sujet André Jardin (1984, p. 448) et Jean-Louis Benoît (2005, pp. 334-335).
} 
de discussion face au despotisme et le texte de son discours ne fut pas reproduit intégralement dans le Recueil des travaux de l'Académie ${ }^{11}$. Pour Boudon, Tocqueville est «d'instinct» le précurseur de ce qu'on appellera plus tard l'individualisme méthodologique (ibid., p.88). Mais, mise en œuvre par Tocqueville, cette méthode centrée sur l'individu et ses raisons d'agir ne néglige pas pour autant les effets émergents. L'agrégation de comportements individuels largement influencés par la structure sociale finit par produire à son tour des effets de structure. Tout cela, chez Tocqueville, est appréhendé par le concept d'état social.

Quelques articles méritent également l'attention. Philippe Steiner, dans les Archives Européennes de Sociologie, examine le fonctionnement de l'héritage égalitaire comme dispositif social (2005, pp. 127-149). Il prend pour point de départ les réflexions de Tocqueville sur l'héritage égalitaire institué par le Code civil. Pour Tocqueville, l'héritage est à la fois «un indicateur de l'état social» et «un dispositif social achevant la marche vers l'égalité sociale». Des lois de succession qui ont pour effet de concentrer la richesse foncière sont à associer à l'aristocratie; dans les sociétés démocratiques au contraire, les lois de succession dispersent cette propriété (Steiner, 2005, pp. 129-130). Mais Steiner montre aussi comment Tocqueville analyse «la dimension causale du dispositif » (ibid., pp. 130 et ss). Dans une étude publiée par la Revue suisse d' histoire à l'occasion du bicentenaire $^{12}$, Marc Vuilleumier analyse la critique présentée par Tocqueville en 1848 du livre De la démocratie en Suisse, publié en 1843 par l'économiste genevois Antoine-Elisée Cherbuliez. Sur plus d'un point - et notamment sur le manque d'indépendance de la justice en Suisse - Tocqueville s'accorde avec Cherbuliez. Il reproche toutefois à ce dernier de considérer la démocratie en Suisse comme établie de longue date, conformément à l'historiographie traditionnelle. Pour Tocqueville, la démocratie suisse est de création toute récente; elle ne s'implanta dans la majorité des cantons qu'à partir de 1830, à l'époque du mouvement de la Régénération (Vuilleumier, 2005, pp. 162-163). Richard Swedberg, dans L'Année sociologique, présente un parallèle intéressant entre Tocqueville et Max Weber. Selon lui, le premier montre aussi bien que le second l'influence du contexte culturel sur le développement du capitalisme. De ce point de vue, De la démocratie en Amérique contient des enseignements qui préfigurent ceux de L'éthique protestante et l' esprit du capitalisme. Le rôle attribué par Tocqueville à la religion dans la vie économique des Etats-Unis peut être comparé à l'impact des idées de Calvin dans la théorie weberienne (2005, pp. 443-444). Dans Le Temps, (8 novembre 2005), Olivier Meuwly reprend la thèse des «institutions de la démocratie» - le fédéralisme, les associations, la religion et le droit - qu'il avait présentée il y a quelques années (2002, chap. 7, pp. 193-206). Ces institutions offrent des principes qui structurent la philosophie de l'Etat de Tocqueville. Mais, estime Meuwly, s'ils ont été de nos jours «élevés comme composants majeurs de la société», ils n'en ont pas moins été «complètement transformés sur le plan lexicologique », ce qui complique singulièrement notre relation à l'œuvre de Tocqueville.

${ }^{11}$ La variante non publiée à l'époque figure dans le Tome XVI des CEuvres complètes parues aux éditions Gallimard, pp. 233-235.

12 Je remercie Giovanni Busino de m'avoir signalé cette parution récente. 
Signalons encore quelques publications récentes. Dans la prestigieuse collection de «La Pléiade», des Euvres choisies de Tocqueville (2004), aux Presses de la Sorbonne, un nouveau livre de Françoise Mélonio en collaboration avec JoséLouis Diaz, Tocqueville et la littérature (2005), et un très utile Sainte-Beuve, Ecrits sur Tocqueville (2004), édité par Michel Brix, qui reproduit les commentaires consacrés à Tocqueville par l'auteur des «Causeries du lundi». Enfin, la Revue Tocqueville/Tocqueville Review a publié un numéro spécial du bicentenaire, édité par Laurence Guellec, sur le thème «Tocqueville et l'esprit de la démocratie » (Vol. XXVI, No 1, 2005) qui reprend un choix d'articles importants publiés précédemment dans la revue. On y trouve notamment des textes de Raymond Aron, François Bourricaud, François Furet, Jean-Claude Lamberti, Claude Lefort, Françoise Mélonio et Mohamed Cherkaoui. Pour sa part, la revue Commentaire a annoncé au printemps 2005 «de nombreuses pages» consacrées «au plus illustre des philosophes politiques français» (No 109, 2005, p. 21). Dans les numéros suivants, priorité fut toutefois donnée par la rédaction à des souvenirs inédits du Comte Hervé de Tocqueville, le père d'Alexis. Pour les aficionados donc.

Dans le genre «grand public», il faut mentionner l'opération BHL en Amérique. A l'invitation de la revue The Atlantic Monthly, Bernard-Henri Lévy a parcouru les Etats-Unis pendant plus d'un an «in the footsteps of Tocqueville». Il en est résulté une série d'articles parus mensuellement à partir de mai 2005 et qui seront publiés prochainement sous le titre American Vertigo chez Random House. Le caractère journalistique de l'entreprise lui a assuré une couverture médiatique tout à fait exceptionnelle aux Etats-Unis. Un critique américain fait toutefois observer que «le sujet réel de Bernard-Henri Lévy n'est pas l'Amérique» mais bien «la représentation française de l'Amérique» (Westbrook, 2005, p. 14).

On peut le constater, le renouveau tocquevillien est effectivement chargé d'ambiguiités. Comme Olivier Meuwly le note ironiquement, «la récupération dont il a été l'objet pourrait d'ailleurs suffire à rendre Tocqueville suspect » (2005, p. 18). Il n'est pas jusqu'à un essayiste médiatique comme Alain Finkielkraut qui ne se sente appelé à mobiliser la vulgate tocquevillienne pour faire dialoguer Tocqueville avec Hannah Arendt et les anciens dissidents de la Charte 77 (Fienkielkraut, 2005, chap. IV, pp. 51-58). La pluralité des préoccupations sousjacentes au «grand retour» de Tocqueville est bien réelle mais elle est due en partie à la personnalité même de ce dernier qui était un homme pétri de contradictions, tantôt porté vers la vision optimiste d'un avenir maîtrisé, tantôt incapable de ne pas céder à la nostalgie d'un passé idéalisé. Il avait une attitude ambivalente à l'égard de la Révolution mais également à l'égard de la société de classes moyennes qui se développait sous ses yeux. Les questions qui l'assaillaient, face à la montée de l'égalitarisme moderne, étaient des questions qui ne recevront sans doute jamais de réponses définitives. Ceci confère une dimension tragique à sa démarche intellectuelle et à sa vie. Il serait regrettable - mais pas tellement improbable - que l'engouement actuel ne contribue à l'instrumentaliser davantage, au service de causes qui n'auraient pas été les siennes.

Université de Genève 


\section{RÉFÉRENCES BIBLIOGRAPHIQUES}

Antoine, Agnès, L'impensé de la démocratie. Tocqueville, la citoyenneté et la religion, Paris, Fayard, 2003.

Aron, Raymond, «Tocqueville retrouvé», Revue Tocqueville/Tocqueville Review, Vol. I, No 1, 1979. Repris dans le numéro spécial du bicentenaire, Vol. XXVI, No 1, 2005.

Audier, Serge, Tocqueville retrouvé. Genèse et enjeux du renouveau tocquevillien français, Paris, Vrin/EHESS, 2004.

Benoît, Jean-Louis, Alexis de Tocqueville, textes essentiels, Paris, Pocket/Agora, 2000.

Benoît, Jean-Louis, Tocqueville moraliste, Paris, Champion, 2004.

Benoît, Jean-Louis, Comprendre Tocqueville, Paris, Armand Colin, 2004.

Benoît, Jean-Louis et Eric Keslassy, Alexis de Tocqueville. Textes économiques, Paris, Pocket/Agora, 2004.

Benoît, Jean-Louis, Tocqueville. Un destin paradoxal, Paris, Bayard, 2005.

Boudon, Raymond, Tocqueville aujourd' hui, Paris, Odile Jacob, 2005.

Coenen-Huther, Jacques, Tocqueville, Paris, PUF, Coll. «Que sais-je?», 1997.

Coenen-Huther, Jacques, Sociologie des élites, Paris, Armand Colin, Coll. «Cursus », 2004.

Finkielkraut, Alain, Nous autres, modernes, Paris, Editions Ellipses, 2005.

Guellec, Laurence, Tocqueville. L'apprentissage de la liberté, Paris, Michalon, 1996.

Guellec, Laurence, Tocqueville et les langages de la démocratie, Paris, Champion, 2004.

Jardin, André, Alexis de Tocqueville 1805-1859, Paris, Hachette, 1984, réimpression «Pluriel», 2005.

Keslassy, Eric, Le libéralisme de Tocqueville à l'épreuve du paupérisme. Paris, L'Harmattan, 2000.

Keslassy, Eric, Question sociale et démocratie dans l' œuvre de Tocqueville, Paris, thèse de doctorat non encore publiée, Université Paris IX-Dauphine, Ecole doctorale de sciences sociales IRISCREDEP, UFR Science des organisations, 2005.

Lévy, Bernard-Henri, «In the Footsteps of Tocqueville», The Atlantic Monthly, sept livraisons à partir de mai 2005.

Lieven, Anatol, America Right or Wrong. An Anatomy of American Nationalism, Londres, HarperCollins Publishers, 2004.

Manzini, Charlotte, Qui êtes-vous Monsieur de Tocqueville? Saint-Lô, Archives départementales de la Manche, 2005.

Mélonio, Françoise, Tocqueville et les Français, Paris, Aubier, 1993.

Mélonio, Françoise et José-Louis Diaz, Tocqueville et la littérature, Paris, Presses de l'Université de Paris-Sorbonne, 2005.

Meuwly, Olivier, Liberté et société. Constant et Tocqueville face aux limites du libéralisme moderne, Genève, Droz, 2002.

Meuwly, Olivier, «Pour être libéral, il ne suffit pas de réduire la surface étatique, il faut repenser l'étendue de l'action de l'Etat», Genève, Le Temps, La vie des idées, mardi 8 novembre 2005, p. 18.

Pierre-Marcel, R., Essai politique sur Alexis de Tocqueville, Paris, Alcan, 1910.

Sainte-Beuve, Charles-Augustin, Ecrits sur Tocqueville. Edition de Michel Brix, Jaignes, La Chasse au Snark, 2004.

Steiner, Philippe, «L'héritage comme dispositif social», Archives Européennes de Sociologie, tome XLVI, No 1, 2005, pp. 127-149.

Swedberg, Richard, «Towards an Economic Sociology of Capitalism», L'Année sociologique, Vol. 55, No 2, 2005, pp. 419-450

The Tocqueville Review/La Revue Tocqueville, Numéro spécial du bicentenaire. Tocqueville et l'esprit de la démocratie (textes réunis par Laurence Guellec), Vol. XXVI, No 1, 2005. 
Tocqueville, De la démocratie en Amérique. Souvenirs. L'Ancien Régime et la Révolution, Introductions et notes de Jean-Claude Lamberti et Françoise Mélonio, Paris, Laffont, Coll. «Bouquins », 1986, réimpressions 1991 et 1999.

Tocqueville, Euvres, Textes présentés par François Furet et Françoise Mélonio, Paris, Gallimard, Coll. «La Pléiade», 2004.

Vuilleumier, Marc, «La Suisse de 1848: 1'analyse de Tocqueville», Revue suisse d' histoire, Vol. 55, No 2, 2005, pp. 149-174.

Westbrook, David A., «Rassurante Amérique. Tocqueville revu par Bernard-Henri Lévy ». Traduit de l'anglais. Esprit, Novembre 2005, pp. 6-21. 